\title{
ESCOLAS E CURSOS DE ENFERMAGEM NA HISTÓRIA DA PROFISSÃO NO BRASIL (1890-1922)
}

Fernando Porto ${ }^{1}$, Wellington de Mendonça Amorim²

'Pós-doutor pela Escola de Enfermagem da Universidade de São Paulo. ${ }^{2}$ Dr. em Enfermagem pela Escola de Enfermagem Anna Nery da UFRJ.

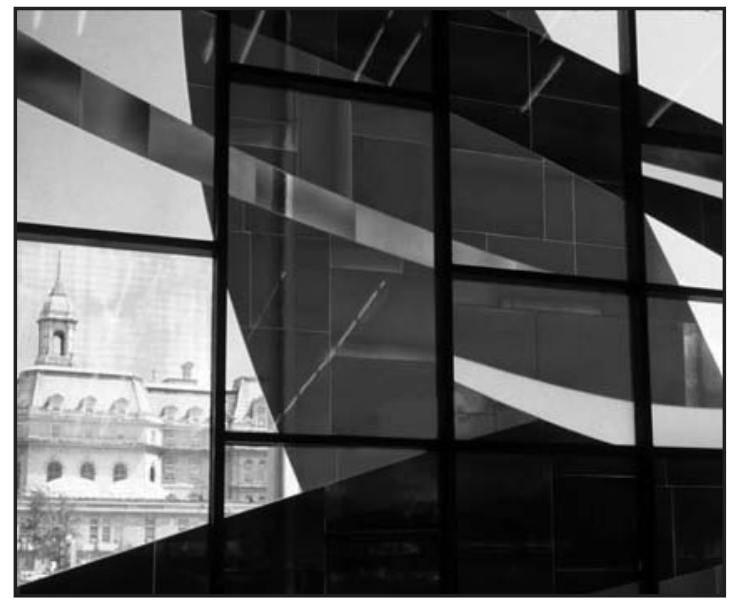

NURSING SCHOOLS AND COURSES IN HISTORY THE PROFESSION IN BRAZIL (1890-1922)

\section{SUMMARY}

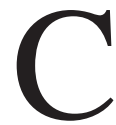

entral approach study, analysis and discussion about the initiatives and embodiments of nursing schools and courses, whose temporary delimitation and geographical $1890-1922$ is the axis Rio de Janeiro and Sao Paulo (Brazil). The theoretical concept was that of professionalism, according to Norbert Elias. Methodologically, the written documents used were originally from the medical press and institutional reports, detailed on the knowledge production of articles, dissertations and theses. The results pointed to a total of seventeen initiatives and embodiments of schools and nursing courses before the introduction of modern nursing in Brazil, through the School of Nursing, Department of Public Health at the beginning of health reform, led by Carlos Chagas.

Key words: Nursing, Nursing History and Education

\author{
ESCUELAS DE ENFERMERÍA Y CURSOS \\ EN LA HISTORIA DE LA PROFESIÓN EN \\ BRASIL (1890-1922)
}

\section{RESUMEN}

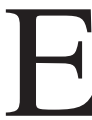
studio de enfoque central, de análisis y discusión en las iniciativas y materializaciones de escuelas y cursos de enfermería, cuya delimitación temporal es 1890-1922 y geográfica, el eje Río de Janeiro y San Paulo (Brasil). La concepción teórica fue la de la profesionalización, según Nobert Elias. Medotológicamente, los documentos escritos utilizados fueron oriundos de la prensa médica e informes institucionales, circunstanciados por la producción del conocimiento de artículos, disertaciones y tesis. Los resultados apuntaron para un total de diecisiete iniciativas y materializaciones de escuelas y cursos de enfermería antes de la implantación de la enfermería moderna en Brasil, por medio de la Escuela de Enfermeras del Departamento Nacional de Salud Pública, a comienzos de la Reforma Sanitaria, liderada por Carlos Chagas.

Palabras-clave: Enfermería, Historia de la Enfermería y Enseñanza

\section{RESUMO}

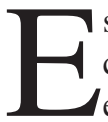
studo de enfoque central de análise e discussão nas iniciativas e materializações de escolas e cursos de enfermagem, na delimitação temporal de 1890-1922 e geográfica no eixo Rio de Janeiro e São Paulo (Brasil). A concepção teórica foi a de profissionalização, segundo Nobert Elias. Medotologicamente, os documentos escritos utilizados foram oriundos da imprensa médica e relatórios institucionais, circunstanciados pela produção do conhecimento de artigos, dissertações e teses. Os resultados apontaram para o total de 
dezessete iniciativas e materializações de escolas e cursos de enfermagem antes da implantação da enfermagem moderna no Brasil, por meio da Escola de Enfermeiras do Departamento Nacional de Saúde Pública, no início da Reforma Sanitária, liderada por Carlos Chagas.

Palavras-chave: Enfermagem, História da Enfermagem e Ensino.

Em 2010, a enfermagem brasileira comemorará 120 anos de profissionalização no Brasil. Essa trajetória, por mais que os pesquisadores se debrucem sobre a massa documental, muito, ainda, se tem a perscrutar. Neste sentido, os resultados das pesquisas apontam para a criação de escolas e cursos de enfermagem, alguns pouco estudados, partiram de iniciativas de profissionalização que o tempo se deu conta de apagar ou se reconfiguraram em virtude do contexto histórico do país.

Há muitos anos a história da enfermagem brasileira foi ensinada aos enfermeiros por meio das obras "Páginas da História da Enfermagem" (1951), a qual com o passar dos anos passou a ser intitulada "História da Enfermagem" (1968), e lida em livro institucional denominado "Séculos de Culturas" no capítulo "A Enfermagem no Rio" publicada pela Universidade do Brasil (1964), atual Universidade Federal do Rio de Janeiro (UFRJ), de autoria da Enfermeira Waleska Paixão, à época, diretora da Escola de Enfermagem Anna Nery, da UFRJ.

O livro "História da Enfermagem" numa perspectiva tradicional da "grande história", remonta às fronteiras da pré-história, onde a Enfermagem era associada ao sentimento de preservação da espécie ou aos instintos maternos para cuidar dos filhos, sendo, possivelmente, o que instiga a novos produtos empreendidos pelos grupos de pesquisa de História da Enfermagem Brasileira, com produção de monografias, dissertações, teses, livros e artigos. Estes estudos caracterizam-se, de modo geral, pelo recorte em linhas temáticas e de objetos históricos, em vez da construção da "Grande História da Enfermagem", em uma perspectiva totalizante em grandes espaços temporais e geográficos comuns às abordagens tradicionais (SILVA JÚNIOR e ALVES, 2006).
Para tanto, o que se apresentará no presente texto é com base na concepção de profissionalização, por meio dos elementos de associação profissional, instrução e conhecimento, e diploma, assim considerado por Nobert Elias (Silva, 1987), sendo, aliás, uma linha de pesquisa, no Laboratório de Pesquisa de História da Enfermagem (LAPHE), da Escola de Enfermagem Alfredo Pinto (EEAP), da Universidade Federal do estado do Rio de Janeiro (UNIRIO), inscrito no Conselho Nacional de Desenvolvimento Científico e Tecnológico (CNPq).

Cabe ressaltar, porém, que a prática dos cuidados de enfermagem, no Brasil, foi realizada por escravos e religiosas, objeto de estudo, pelo menos, por duas teses de doutorado, a saber: "A mística do silêncio - a enfermagem na Santa Casa de Misericórdia do Rio de Janeiro no século XIX" de autoria da pesquisadora Maria Itayra Coelho de Souza Padilha (1998) e "A ordem no hospício: primórdios da enfermagem psiquiátrica no Brasil (1852-1890)“ de Maria Angélica de Almeida Peres (2008) ambos desenvolvidos no âmbito do Núcleo de Pesquisa em História da Enfermagem Brasileira (NUPHEBRAS), da Escola de Enfermagem Anna Nery, da UFRJ.

Mediante o exposto e a abrangência da temática sobre a História da Enfermagem no Brasil, é mister a delimitação temporal inicialmente enunciada nos estudos históricos brasileiros no ano de 1890, com a criação da Escola Profissional de Enfermeiros e Enfermeiras da Assistência a Alienados, atual Escola de Enfermagem Alfredo Pinto da UNIRIO, por meio do Decreto 791, quando era anexada ao Hospício Nacional de Alienados, localizado no Rio de Janeiro, como a primeira instituição de ensino na enfermagem. O marco final, em 1922, com a criação da Escola de Enfermeiras do Departamento Nacional de Saúde Pública, também reconhecida, por meio do modelo norte-americano, com a instituição de ensino que implantou a enfermagem moderna, atual Escola de Enfermagem Anna Nery da Universidade Federal do Rio de Janeiro.

Ademais, a delimitação espacial se dá no Rio de Janeiro, como capital do Brasil à época, e a cidade de São Paulo, por ter atraído na virada do século XIX para o XX capitalistas e fazendeiros no 


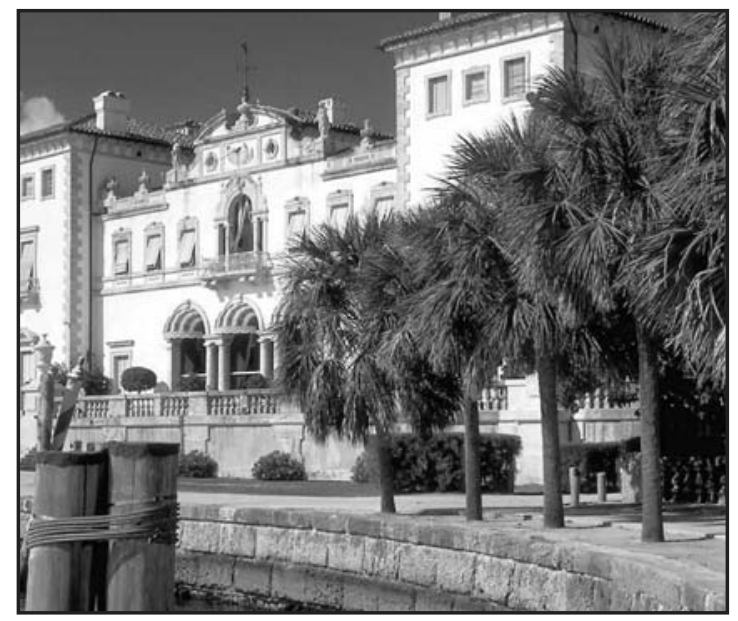

sentido do crescimento econômico do país no período republicano.

\section{REPÚBLICA E ENFERMAGEM}

O Brasil passa do Regime Monárquico para o Regime Republicano, em 1889. Nessa transição a historiadora e antropóloga, Angela Marques da Costa e Lilia Mortiz Schwarcz (2000), supõe que a República representou a modernidade que se instalava no país, tirando o país da letargia ou barbárie da escravidão - período monárquico -, sendo entendida como uma batalha simbólica, quando nomes, hinos, bandeiras, heróis e modelos foram substituídos ou alterados com o intuito de marcar a diferença entre os dois regimes.

Nesse contexto, ocorreu a laicização da enfermagem no Brasil, quando as Irmãs da Caridade da Santa Casa de Misericórdia do Rio de Janeiro saem do Hospício Nacional de Alienados e, em síntese, pelo contexto histórico e necessidade de recursos humanos institucional, dão assistência aos alienados da instituição, quando se deu a criação da Escola Profissional de Enfermeiros e Enfermeiras.

Os estudos de Almerinda Moreira e Tiago Braga do Espírito Santo (2010, 2007) evidenciaram que o ensino da enfermagem, naquela instituição, se deu sob a influência do modelo francês de Bourniville, lembrando que a França, com Felipe Phinel, modernizava a assistência psiquiátrica.

Entre os anos de 1890 a 1922, iniciativas e materializações de escolas e cursos de enfermeiras no Rio de Janeiro e São Paulo ocorreram no con- texto sócio histórico e sanitário, em síntese, abarcaram a Febre Amarela (1903), I Guerra Mundial (1914-1918), Gripe Espanhola (1918-1919) e o início da Reforma Sanitária (1920), liderada por Carlos Chagas como circunstancias para o desenvolvimento da enfermagem no Brasil.

A historiadora Maria Lúcia Mott, no estudo intitulado "Revendo a história da enfermagem em São Paulo (1890-1920)" (1999), relata que, em 1894, o Curso de Enfermeiras do Hospital Samaritano, em São Paulo, foi criado com a presença de Enfermeiras Inglesas, tendo apenas aprendizes do sexo feminino, quando formou profissionais à época. Dois anos depois (1896), no Hospital de Isolamento outra iniciativa, por meio do Decreto n. 412, previa o funcionamento de Curso para Enfermeiras e em meados dos anos de 1906 foi proposto pela Congregação de Farmácia, pelo dr. Valeriano de Souza, a criação de uma Escola de Enfermeiros, porém, ambas não saíram do papel. Ainda, em 1906, a Santa Casa de Misericórdia identificou a necessidade da criação de uma Escola de Enfermeiras, que funcionou de 1912 até 1917. Outro Curso de Enfermeiras, criado em São Paulo, foi o da Maternidade de São Paulo, em 1908, voltado para os cuidados obstétricos, tendo funcionado até 1913 , de acordo com os relatórios da diretoria institucional (Mott, 1999).

Em 1908, foi criada a Cruz Vermelha Brasileira no Rio de Janeiro e, em 1912 em São Paulo, a Médica Maria Rennotte criou o Curso de Enfermeiras da Cruz Vermelha. No Rio de Janeiro, em 1912, tem-se o Curso de Damas da Cruz Vermelha Brasileira; em 1914, o Curso de Enfermeiras Voluntárias e, em 1916, o Curso de Enfermeiras Profissionais e a criação da Escola Prática de Enfermeiras da Cruz Vermelha Brasileira tendo como Diretor o Médico Getúlio dos Santos (Histórico da Cruz Vermelha Brasileira, 1923; Mott, 1999 e Porto e Santos, 2008).

No ano de 1917, a Policlínica de Botafogo, no Rio de Janeiro, criou o Curso de Enfermeiras, o qual, até 1920 , formou duas turmas de enfermeiros, segundo o estudo da historiadora Maria Lúcia Mott. Ademais, no discurso de inauguração deste curso o Dr. Ernani Lopes - Diretor do Hospício Nacional de Alienados - citou a materialização da Escola Profissional de Enfermeiros e Enfermeiras 
e da Escola Prática de Enfermeiras da Cruz Vermelha Brasileira e destacou que, caberia naquele momento registrar que:

"no Rio varias instituições hospitalares que instruem e formam os seus enfermeiros. Taes são: a Maternidade das Laranjeiras, O Posto Central da Assistência , o Hospital de Marinha (Dr PortoCarrero) e, particularmente, a Santa Casa de Misericórdia." (Curso de Enfermeiras da Policlínica de Botafogo, 1919, 49).

O Curso de Enfermeiras da Assistência Particular de Nossa Senhora da Gloria, foi veiculado na imprensa médica, em 1920, com citação em seu Estatuto do artigo terceiro, sobre a criação de uma Escola de Enfermeiras "Anexo aos serviços hospitalares creará um curso de enfermeiras, ampliando e creando tantos estabelecimentos e departamentos quantos lhe permitam os recursos." Ainda, segundo o mesmo registro, a justificativa de sua criação era "(...) falta dessas profissionais, competentes, constitue inegavelmente a maior dificuldade com que lutam os diretores dos nossos hospitais e casas de saúde." (Revista BrazilMedico, 1920, 26).

Em 1920, alguns dias após a posse do novo Prefeito do Rio de Janeiro, o Sr Carlos César Oliveira Sampaio, se vê na imprensa médica a divulgação do Curso de Enfermeiros do Posto Central de Assistência, o qual foi organizado através de uma comissão nomeada pelo Diretor de Higiene Dr Luiz Pedro Barbosa - Diretor-geral do Departamento Municipal de Assistência Pública (1920) e Diretor da Policlínica de Botafogo desde 1901.

O curso era composto pelos médicos: Gastão Guimarães, Girondino Esteves e Marques Canário, que estabeleceram as disposições gerais de cinco artigos sob os títulos de "Do Curso" "Da matricula", "Da freqüência", "Do pessoal" e "Provas", cada qual com os seus respectivos artigos e ao final a apresentação das matérias a serem abordadas no curso, como: Noções de Anatomia e Fisiologia, Noções de Higiene, Cuidados médicos de higiene, Cuidados cirúrgicos aos doentes e cuidados aos feridos, que destacamos aqui o primeiro artigo:
"Do Curso - 10 O curso de enfermeiros anexo ao Posto Central de Assistência tem por fim o ensino permanente dos enfermeiros de ambos os sexos que trabalham nas repartições subordinadas à Diretoria de Assistência Municipal." (Revista Brazil-Medico, 1920, 560)

Dias depois, outra iniciativa de profissionalização é anunciada pela imprensa médica, a Escola de Enfermeiras Municipais:

"Estava marcada para quarta-feira última, 29 de setembro a inauguração da Escola de Enfermeiras, organizada pela Diretoria de Assistencia. O corpo docente ficou constituido pelos Srs Drs Lafayette de Souza, Diretor do curso, Pedro Paulo de Carvalho, cadeira de higiene e cuidados de assistência aos doentes; Marques Canario, clinica médica; e J. Belesa anatomia e fisiologia." (Revista Brazil-Medico, 1920, 661)

Mesmo diante das iniciativas de profissionalização da enfermagem, a imprensa médica divulgava que:

“(...) não nos capacitamos no Brazil de que o mister de enfermeiros exige uma certa cultura de espirito, indispensável para acquisição do preparo profissional. O enfermeiro preciza ser um auxiliar do medico, capaz de comprehender-lhe as prescripções e executa-las conscientemente e com plena responsabilidade. Entre nós, sobretudo nos grandes hospitaes, além de desprovidos de preparo propriamente profissional, são os enfermeiros indivíduos bisonhos, incultos, na maioria dos casos quasi analphabetos." (Revista BrazilMedico, 1920, 312).

A partir de 1920, muitos são os estudos sobre a Reforma Sanitária e a implantação da enfermagem moderna, por meio da criação da Escola de Enfermeiras do Departamento Nacional de Saúde Pública, mas as iniciativas e materializações às vezes careceram de serem consideradas.

A assertiva se pauta nas palavras da responsável pela implantação da enfermagem moderna no Brasil, a Enfermeira Norte-americana Ethel Parsons ao ter afirmado em seu relatório que "Não 
havendo, no Brasil, escolas de enfermagem com o preparo adequado para esse trabalho ..." veiculado em fac-simile pela Revista de Enfermagem Escola Anna Nery (1997).

Por outro lado, pode-se entender a assertiva de Parsons no sentido que, ela teria se referido, provavelmente, a abordagem do atendimento da enfermagem direcionada à saúde pública, na visão do modelo norte-americano, que à época desejava se realinhar aos moldes propostos no Relatório Goldmark, conforme destacou o estudo intitulado "A Enfermagem de Saúde Pública no Distrito Federal: a influência do Relatório Goldmark (1923 a 1927)" de autoria de Mary Ann Menezes Freire e Wellington Mendonça de Amorim.

Destarte, ela não estaria equivocada, apesar de em seu relatório omitir a ausência das iniciativas e materializações existentes, exemplo disso, a Escola Profissional de Enfermeiros e Enfermeiras e da Escola Prática da Cruz Vermelha Brasileira. Mas isto para se entender é necessário se aprofundar mais na história da enfermagem brasileira.

O contexto sócio-histórico e sanitário nas décadas de 1900, 1910 e início de 1920 exigiu medidas que impulsionaram muitas iniciativas e materializações de escolas e cursos de enfermagem, que hoje, reconfiguradas, pode-se citar que a Escola de Enfermagem Alfredo Pinto tenha vencido períodos difíceis, mas a persistência no ensino em prol da enfermagem tenha-se chegado até onde chegamos.

Mediante ao exposto, em 2010, o Brasil completará 120 anos de profissionalização de enfermagem, por meio da Escola de Enfermagem Alfredo Pinto da Universidade Federal do Estado do Rio de Janeiro, isto significa comemorar a luta da enfermagem brasileira como profissão.

\section{CONSIDERAÇÕES FINAIS}

Atualmente no Brasil tem-se, aproximadamente, 550 Cursos de Enfermagem inseridos nas Universidades como indicador do resultado da trajetória da profissionalização da enfermagem no país.

Neste sentido, as entidades de classes tiveram a sua importância no processo de profissionalização da enfermagem. A primeira é denominada Associação Brasileira de Enfermagem (ABEn), criada em 1926; anos mais tarde a criação do siste- ma Conselho Regional de Enfermagem (COREn) e Conselho Federal de Enfermagem (COFEn), em 1973, para a fiscalização do exercício profissional; depois, o Sindicato de Enfermeiros por unidade da federação, criados a partir da década de 1970, repercutindo na criação da Federação Nacional dos Enfermeiros e a pulverização das Associações de Especialistas.

Por outro lado, remontar a história da enfermagem, pelos eixos temáticos apresentados no I Simpósio Ibero-americano da História em Enfermagem, realizado em São Paulo em 2007, como a História das Instituições, entende-se como uma das maneiras de manutenção para se produzir conhecimento, mas, também, que seja o momento da enfermagem brasileira avançar nos eixos de Historia de Vida e Antropologia dos Cuidados, por meio dos Programas de Pós-graduação (Mestrado e Doutorado), instituídos, inicialmente no eixo Rio-São Paulo, nas décadas de 1970 e 1980, que conta com 35 Programas stricto senso distribuídos pelo Brasil, reconhecidos pela Coordenação de Aperfeiçoamento de Pessoal de Nível Superior (CAPEs) e de aproximadamente 373 Grupos de Pesquisa, totalizando, em torno de 32 com o descritor "História da Enfermagem" inscritos no CNPq.

Estes dados podem contribuir para se averiguar o desenvolvimento da enfermagem no país, inclusive, como foi possível de observar, com a presença, minoritária, mas presente do gênero masculino na profissão.

\section{REFERÊNCIAS}

- Brazil-Medico. (10/01/1920). Assistência Particular Nossa Senhora da Glória. Revista Brazil-Medico. Rio de Janeiro (Brasil). XXIV (2): 26.

- Brazil-Medico. (15/05/1920) Escola para Enfermeiros. Revista Brazil-Medico. Rio de Janeiro (Brasil). XXIV (20): 312.

- Brazil-Medico. (21/08/1920) .Diretoria de Higiene e Assistência Pública. Revista Brazil-Medico. Rio de janeiro (Brasil) XXIV (34): 560.

- Brazil-Medico. (02/101920). Escola de Enfermeiras Municipais. Revista Brazil-Medico. Rio de Janeiro (Brasil) XXIV(40): 661.

- Brazil-Medico. (02/10/1920) Escola de Enfermeiras Municipaes - Notas e Informações. Revista Brazil-Medico. Rio de Janeiro XXIV (40): 661.

- Costa, AM e Schwarcz, LM (2000). Virando o século - 18901914 no tempo das certezas. São Paulo (Brasil). Companhia das Letras. 
- Cruz Vermelha Brasileira. (1923). Histórico da Cruz Vermelha Brasileira (1908-1923). Rio de Janeiro (Brasil): Órgão Central. Cruz Vermelha.

- Espirito Santo, TB. (2007). Enfermeiras francesas na capital do Brasil (1890-1895) [dissertação de mestrado]. São Paulo (Brasil). Escola de Enfermagem da Universidade de São Paulo. Universidade de São Paulo.

- FREIRE, MAM e Amorim, WMA. (2008). Enfermagem de Saúde Pública no Distrito Federal: A Influência do Relatório Goldmark (1923 a 1927). Rio de Janeiro (Brasil). Escola Anna Nery. Revista de Enfermagem 12 (1):115-124.

- Mott, ML. (1999). Revendo a história da enfermagem em São Paulo (1890-1920). Cadernos Pagu. São Paulo (Brasil).13: 327-355.

- Moreira, A. (2010). A origem da enfermagem brasileira. História da Enfermagem - versões e interpretações. 3a Edição. Rio de Janeiro (Brasil). Revinter:.81-141.

- Parsons, E (1997). A enfermagem moderna no Brasil (facsímiles). Revista de Enfermagem Escola Anna Nery. Rio de Janeiro (Brasil) 1: 9-24.

- Padilha, MICS. (1998). A Mística do Silêncio - a enfermagem na Santa Casa de Misericórdia do Rio de Janeiro no século XIX. Pelotas (Brasil). Ed. Universitária/UPel.
- Peres, MAA. (2008). A ordem no hospício: primórdios da enfermagem psiquiátrica no Brasil (1852-1890)[tese de doutorado. Rio de Janeiro (Brasil). Universidade Federal do Rio de Janeiro. Escola de Enfermagem Anna Nery.

- Porto, F e Santos, TCF. (2008). A Enfermeira brasileira na mira do clik fotográfico (1919-1925). In; História da Enfermagem - lutas, ritos e emblemas. Porto, F. Amorim. W. (organizadores). Rio de Janeiro (Brasil): Águia Dourada: 25188.

- Policlínica de Botafogo. (1919). Histórico do Curso de Enfermeiras da Policlínica de Botafogo. Imprensa Oficial. Rio de Janeiro (Brasil).

- Silva, B. (1987). Dicionário de Ciências Sociais. Verbete de profissões autoria Nobert Elias. Fundação Getulio Vargas. Instituto de Documentação. Coordenação Geral. Rio de Janeiro (Brasil): 993-994.

- Silva Júnior, OC e Alves, APC. (2006). Páginas de História da Enfermagem: o jubileu de ouro de uma obra (1951-2001).

- Esc. Anna Nery 10 (2). Rio de Janeiro (Brasil). Disponível em: http://www.portalbvsenf.eerp.usp.br/scielo.php?script= sci_arttext\&pid=S1414-81452006000200003\&lng=pt\&nrm =iso. Capturado em 17 de janeiro de 2009.

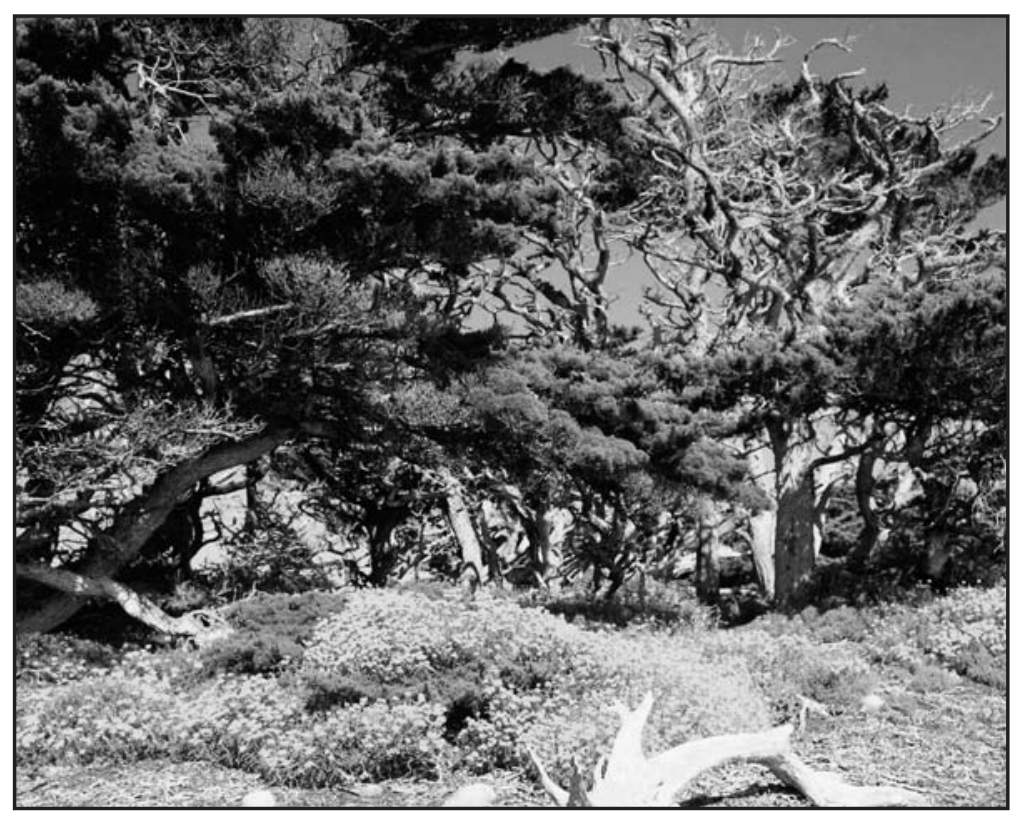

